\title{
Real Power Loss Reduction by Melon Fly Optimization and Spontaneous Process Algorithm's
}

\author{
Lenin Kanagasabai ${ }^{*}$ \\ https://orcid.org/0000-0002-8989-2441 \\ Editor-in-Chief: Alexandre Rasi Aoki \\ Associate Editor: Alexandre Rasi Aoki \\ Received: 2020.07.31; Accepted: 2021.03.20. \\ *Correspondence: gklenin@gmail.com; Tel.: +91-9080574470 (L.K.).
}

${ }^{1}$ Prasad V.Potluri Siddhartha Institute of Technology, Department of EEE, Kanuru, Vijayawada, Andhra Pradesh, India.

\section{HIGHLIGHTS}

- Melon Fly algorithm based on the innate events of Melon fly

- By smell and vision the Melon fly will move to the best location

- Spontaneous Process Algorithm based onnuclear fission and fusion

- Nucleus symbolizes the variables and potential solution

\begin{abstract}
In this work Melon Fly Optimization (MFO) Algorithm and Spontaneous Process Algorithm (SPA) is designed to reduce the Real power loss, voltage stability enhancement and reducing the Voltage deviation. In this work real power loss measured and how much loss has been reduced is also identified by suitable comparison with standard algorithms. In this society from common consumer to industry needs better quality of power continuously and constantly without much variation. One way to improve the quality of the power is to reduce the power loss. Also reduction of power loss will improve the economic conditions of the nation indirectly and it improves the productivity of the nation with any hurdles. Around the world all nations sequentially identifying the method to reduce the power loss in the transmission and subsequently it improve the quality of power. MFO algorithm has been formed based on the innate events of Melon fly. Due their very excellent eyesight and mutual supportive behaviour Melon fly will find the food without difficulty. By smell and vision the Melon fly will move to the best location form the current location. In the preliminary level Melon flies will search the food in multiple directions and they may be far away from the food source, it like scattering in the plane. Then Spontaneous Process Algorithm (SPA) is designed to solve the optimal reactive power problem Formulation of the projected algorithm is done by imitating the process done during nuclear fission and fusion. Every item of a nucleus attribute symbolizes each solution variable. Sequence of operators directs the nucleus and in order to avoid the local optimum it will imitate the dissimilar condition of reaction. In the exploration space nucleus symbolizes the variables and potential solution. Levy flight has been intermingled in the procedure to enhance the diversification and intensification in the search. Evaluation of validity of the Melon Fly Optimization (MFO) Algorithm and Spontaneous Process Algorithm (SPA) is done in IEEE 30-bus system by considering voltage stability ( $L$-index) and also devoid of L-index criterion. Minimization of voltage deviation, voltage stability enhancement and power loss minimization has been achieved.
\end{abstract}

Keywords: Optimal Reactive Power; Transmission Loss;Melon Fly Optimization Algorithm; Spontaneous Process Algorithm. 


\section{INTRODUCTION}

Power loss minimization, voltage stability enhancement and deviation of voltage minimization are multiple objectives of this work. Many countries are trying to identify the way to improve the quality of power. Yet many technical obstacles are found in the process. Reduction of power loss is key factor to improve the quality of power in the network. Since from common (small) consumers to high capacity consumers need better quality of power continuously. It will improve the productivity of the nation and economically it will augment the financial strength of the nation. So in this work two algorithms defined and designed for power loss reduction with voltage stability enhancement. Different conventional methods [1-6] and various Evolutionary algorithms [7-10] are utilized to solve the Science and Engineering problems. Main concern is reaching the optimal solution [22-25]. In the conventional methods due to the constraints many techniques failed to reach the better solution. In the swarm and other evolutionary algorithms the major problem is balancing the exploration and exploitation. Since when both are not balanced then reaching the optimal solution will not be possible. In this work Melon Fly Optimization (MFO) Algorithm and Spontaneous Process Algorithm (SPA) has been designed to balance the exploration and exploitation sequentially. At first in this paper Melon Fly Optimization (MFO) Algorithm applied to solve the optimal reactive power problem. Proposed algorithm has been modelled based on the natural actions of Melon fly. Every Melon fly maintain pathway from its position and keep the attention towards food. Dissimilar types of Smell are compared, and the largest food region will be chosen. After the completion of definite number of iterations' the melon fly will not fly arbitrarily with the obtained memory so a memory move or progress direction has been applied in the algorithm. Proposed MFO algorithm improves the search to reach the global optimal solution and also evade the local optima. Then in this paper Spontaneous Process Algorithm (SPA) a physics-based algorithm which has been inspired by nuclear reaction is applied to solve the optimal reactive power problem. Process of Nuclear fission and fusion are utilized to formulate the algorithm. In order to explore the nearby area positions and also to control the capability of exploration and exploitation " $\beta$ " decay will be emulated by Gaussian walk. Getting trapped in Local optimal solution is avoided by applying the Levy flight. In ionization phase, all nucleuses are categorized through fitness values. With and without considering L-index Melon Fly Optimization (MFO) Algorithm and Spontaneous Process Algorithm (SPA) evaluated in IEEE 30 bus system. Deviation of voltage minimization, power loss reduction and voltage stability augmentation achieved. With other reported standard algorithms - Hybrid PSO-Tabu search, Ant lion, quasi-oppositional teaching learning based, improved stochastic fractal search optimization algorithm, harmony search, improved pseudogradient search particle swarm optimization, cuckoo search algorithm, Evolutionary programming and self adaptive real coded genetic algorithm. Projected algorithms MFO and SPA minimized the power loss in both with and without considering voltage stability. Then the percentage of power loss reduction also improved substantially.

\section{Problem Formulation}

In recent years, the problem of voltage stability and voltage collapse has become a major concern in power system planning and operation. To enhance the voltage stability, voltage magnitudes alone will not be a reliable indicator of how far an operating point is from the collapse point. Voltage stability evaluation using modal analysis is used as the indicator of voltage stability.

Objective function of the problem is mathematically defined in general mode by,

$$
\text { Minimization } \tilde{F}(\bar{x}, \bar{y})
$$

Subject to

$$
\begin{aligned}
& E(\bar{x}, \bar{y})=0 \\
& I(\bar{x}, \bar{y})=0
\end{aligned}
$$

Minimization of the Objective function is the key and it defined by " $F$ ". Both $E$ and I indicate the control and dependent variables of the optimal reactive power problem (ORPD). "x" consist of control variables which are reactive power compensators $\left(Q_{c}\right)$, dynamic tap setting of transformers -dynamic $(\mathrm{T})$, level of the voltage in the generation units $\left(V_{g}\right)$.

$x=\left[V G_{1}, \ldots, V G_{N g} ; Q C_{1}, \ldots, Q C_{N C} ; T_{1}, \ldots, T_{N_{T}}\right]$

"y" consist of dependent variables which has slack generator $P G_{\text {slack }}$, level of voltage on transmission lines $V_{L}$, generation units reactive power $Q_{G}$, apparent power $S_{L}$. 
$y=\left[P G_{\text {slack }} ; V L_{1}, \ldots, V L_{N_{\text {Load }}} ; Q G_{1}, \ldots, Q G_{N g} ; S L_{1}, \ldots, S L_{N_{T}}\right]$

Then the single objective problem formulation is defined as follows.

The fitness function $\left(O F_{1}\right)$ is defined to reduce the power loss $(\mathrm{MW})$ in the system is written as,

$O F_{1}=P_{\text {Min }}=\operatorname{Min}\left[\sum_{m}^{N T L} G_{m}\left[V_{i}^{2}+V_{j}^{2}-2 * V_{i} V_{j} \cos \emptyset_{i j}\right]\right]$

Number of transmission line indicated by "NTL", conductance of the transmission line between the $i^{\text {th }}$ and $j^{\text {th }}$ buses, phase angle between buses $\mathrm{i}$ and $\mathrm{j}$ is indicated by $\emptyset_{i j}$.

Minimization of Voltage deviation fitness function $\left(\mathrm{OF}_{2}\right)$ is given by,

$O F_{2}=\operatorname{Min}\left[\sum_{i=1}^{N_{L B}}\left|V_{L k}-V_{L k}^{\text {desired }}\right|^{2}+\sum_{i=1}^{N g}\left|Q_{G K}-Q_{K G}^{L i m}\right|^{2}\right]$

Load voltage in $k^{\text {th }}$ load bus is indicated by $V_{L k}$, voltage desired at the $k^{\text {th }}$ load bus is denoted by $V_{L k}^{\text {desired }}$, reactive power generated at $k^{\text {th }}$ load bus generators is symbolized by $Q_{G K}$, then the reactive power limitation is given by $Q_{K G}^{L i m}$, then the number load and generating units are indicated by $N_{L B}$ and $N g$.

Then the voltage stability index (L-index) fitness function $\left(\mathrm{OF}_{3}\right)$ is given by,

$O F_{3}=\operatorname{Min} L_{\operatorname{Max}}$

$L_{\text {Max }}=\operatorname{Max}\left[L_{j}\right] ; j=1 ; N_{L B}$

And $\left\{\begin{array}{c}L_{j}=1-\sum_{i=1}^{N P V} F_{j i} \frac{V_{i}}{V_{j}} \\ F_{j i}=-\left[Y_{1}\right]^{1}\left[Y_{2}\right]\end{array}\right.$

Such that

$$
L_{\text {Max }}=\operatorname{Max}\left[1-\left[Y_{1}\right]^{-1}\left[Y_{2}\right] \times \frac{V_{i}}{V_{j}}\right]
$$

The objectives of the reactive power dispatch problem considered here is to diminish the system real power loss and maximize the static voltage stability margins (SVSM). This objective is accomplished by appropriate alteration of reactive power variables like generator voltage magnitude, reactive power generation of capacitor bank, and transformer tap setting. Power flow equations are the equality constraints of the problem, while the inequality constraints comprise of the limits on real and reactive power generation, bus voltage magnitudes, transformer tap positions and line flows.

Then the equality constraints are

$$
\begin{aligned}
& 0=P G_{i}-P D_{i}-V_{i} \sum_{j \in N_{B}} V_{j}\left[G_{i j} \cos \left[\emptyset_{i}-\emptyset_{j}\right]+B_{i j} \sin \left[\emptyset_{i}-\emptyset_{j}\right]\right] \\
& 0=Q G_{i}-Q D_{i}-V_{i} \sum_{j \in N_{B}} V_{j}\left[G_{i j} \sin \left[\emptyset_{i}-\emptyset_{j}\right]+B_{i j} \cos \left[\emptyset_{i}-\emptyset_{j}\right]\right]
\end{aligned}
$$

Where, $n b$ is the number of buses, $P_{G}$ and $Q_{G}$ are the real and reactive power of the generator, $P_{D}$ and $Q_{D}$ are the real and reactive load of the generator, and $G_{i j}$ and $B_{i j}$ are the mutual conductance and susceptance between bus iand bus $j$.

Inequality constraints

$$
\begin{gathered}
\mathrm{P}_{\mathrm{gslack}}^{\text {minimum }} \leq \mathrm{P}_{\text {gslack }} \leq \mathrm{P}_{\text {gslack }}^{\text {maximum }} \\
\mathrm{Q}_{\mathrm{gi}}^{\text {minimum }} \leq \mathrm{Q}_{\mathrm{gi}} \leq \mathrm{Q}_{\mathrm{gi}}^{\text {maximum }}, \mathrm{i} \in \mathrm{N}_{\mathrm{g}}(14) \\
\mathrm{VL}_{\mathrm{i}}^{\text {minimum }} \leq \mathrm{VL}_{\mathrm{i}} \leq \mathrm{VL}_{\mathrm{i}}^{\text {maximum }}, \mathrm{i} \in \mathrm{NL} \quad(16) \\
\mathrm{T}_{\mathrm{i}}^{\text {minimum }} \leq \mathrm{T}_{\mathrm{i}} \leq \mathrm{T}_{\mathrm{i}}^{\text {maximum }}, \mathrm{i} \in \mathrm{N}_{\mathrm{T}} \quad(17) \\
\mathrm{Q}_{\mathrm{c}}^{\text {minimum }} \leq \mathrm{Q}_{\mathrm{c}} \leq \mathrm{Q}_{\mathrm{C}}^{\text {maximum }}, \mathrm{i} \in \mathrm{N}_{\mathrm{C}}(18) \\
\left|S L_{i}\right| \leq S_{L_{i}}^{\text {maximum }}, \mathrm{i} \in \mathrm{N}_{\mathrm{TL}} \quad(19) \\
\mathrm{VG}_{\mathrm{i}}^{\text {minimum }} \leq \mathrm{VG}_{\mathrm{i}} \leq \mathrm{VG}_{\mathrm{i}}^{\text {maximum }}, \mathrm{i} \in \mathrm{N}_{\mathrm{g}}
\end{gathered}
$$

Multi objective fitness (MOF) function has been defined by, 


$$
\begin{array}{r}
M O F=F_{1}+r_{i} F_{2}+u F_{3}=F_{1}+\left[\sum_{i=1}^{N L} x_{v}\left[V L_{i}-V L_{i}^{\text {min }}\right]^{2}+\sum_{i=1}^{N G} r_{g}\left[Q G_{i}-Q G_{i}^{\text {min }}\right]^{2}\right]+r_{f} F_{3} \\
V L_{i}^{\text {minimum }}=\left\{\begin{array}{c}
V L_{i}^{\text {max }}, V L_{i}>V L_{i}^{\text {max }} \\
V L_{i}^{\text {min }}, V L_{i}<V L_{i}^{\text {min }}
\end{array}\right. \\
Q G_{i}^{\text {minimum }}=\left\{\begin{array}{c}
Q G_{i}^{\text {max }}, Q G_{i}>Q G_{i}^{\text {max }} \\
Q G_{i}^{\text {min }}, Q G_{i}<Q G_{i}^{\text {min }}
\end{array}\right.
\end{array}
$$

Where, nc, ng and nt are numbers of the switchable reactive power sources, generators and transformers. The equality constraints are satisfied by running the power flow program. The active power generation $\left(\mathrm{P}_{\mathrm{gi}}\right)$, generator terminal bus voltages $\left(\mathrm{V}_{\mathrm{gi}}\right)$ and transformer tap settings $\left(\mathrm{t}_{\mathrm{k}}\right)$ are the control variables and they are self-restricted by the optimization algorithm. The active power generation at slack bus $\left(P_{s 1}\right)$, load bus voltage $\left(V_{\text {load }}\right)$ and reactive power generation $\left(Q_{\mathrm{gi}}\right)$ are the state variables and are restricted by adding a quadratic penalty term to the objective function.

\section{Melon Fly Optimization Algorithm}

In this work Melon Fly Optimization (MFO) Algorithm has been modelled based on the normal events of Melon fly. Because of outstanding eyesight and reciprocated helpful behaviour Melon fly will find the food devoid of complicatedness. Each Melon fly preserve a lane from its position and concentration will be towards the food. Dissimilar types of Smell are evaluated, and the major food region will be preferred. Subsequently the key concentration is indicated as the most excellent present location. By smell and vision the Melon fly will move to the most excellent location form the existing location. Proposed MFO algorithm perk up the search to attain the global optimal solution and also avoid the local optima.

$$
\text { Minimum fitness }(Q) \text { s.t } y_{j} \in\left[\text { Lower bound }_{j}, \text { Upper bound }_{j}\right], j=1,2 ., 3, \ldots, n
$$

Concentration of smell in the Melon fly optimization is the reciprocal of the objective functionfitness $(Q)$; $Q=\left(q_{1}, q_{2}, \ldots, q_{n}\right)$ is the vector of the melon flies.

Parameters are initialized then the preliminary location of the swarm is defined by,

$\left\{\begin{array}{l}Q_{\text {initial }}=\text { Lower bound }+(\text { Upper bound }- \text { Lower bound }) * \text { arbitrary }() \\ P\end{array}\right.$

By the property of smell Melon fly explore the food and it will share the information with co- melon flies.

New-fangled location of the Melon fly is engendered by,

$\left\{\begin{array}{l}Q_{i}=Q_{\text {initial }}+\operatorname{arbitrary}() \\ P_{i}=P_{\text {initial }}+\operatorname{arbitrary}()\end{array}\right.$

Distance is computed from the preliminary location

Distance $_{i}=\sqrt{Q_{i}^{2}+P_{i}^{2}}$

Judgment value $\left(J v_{i}\right)$ of the smell concentration in Melon fly is reciprocal of the distance. Smell concentration (smell concentration ${ }_{i}$ ) is obtained by,

$$
\left\{\begin{array}{c}
J v_{i}=1 / \text { Distance }_{i} \\
\text { smell concentration } \left._{i}=\text { fitness }_{(}\right)
\end{array}\right.
$$

Pick the minimum smell concentration value from melon flies and movement with respect to location defined by,

$$
\left\{\begin{array}{c}
{[\text { Top smell, Top index }]=\text { Minimum }(\text { smell })} \\
\left\{\begin{array}{l}
Q_{\text {initial }}=Q(\text { Top } \text { index }) \\
P_{\text {initial }}=P(\text { Top } \text { index })
\end{array}\right.
\end{array}\right.
$$

Searching the food in several directions in the early stages and sometimes melon fly may move away from the food source, (scattering in the plane) and it defined by, 
In beginning phase the food source may be small,

$$
\left\{\begin{array}{c}
\left\{\begin{array}{c}
Q_{\text {initial }}=\operatorname{arbitrary}(\text { domain }) \\
P_{\text {initial }}=\operatorname{arbitrary}(\text { domain })
\end{array}\right. \\
{\text { Location } \text { initial stage }_{\text {in }}}=\left\{\begin{array}{c}
\sqrt{Q_{i}^{2}+P_{i}^{2}} \text { if } \mathrm{Q}_{\mathrm{i}}+\mathrm{P}_{\mathrm{i}}>0 \\
-\sqrt{Q_{i}^{2}+P_{i}^{2}} \text { if } \mathrm{Q}_{\mathrm{i}}+\mathrm{P}_{\mathrm{i}}<0
\end{array}\right. \\
\text { Top smell } \text { initial stage }=\text { Fitness }\left(\text { Location }_{\text {initial stage }}\right)
\end{array}\right.
$$

$$
\left\{\begin{array}{l}
\left\{\begin{array}{l}
Q_{t}(i)=Q_{\text {initial }}(i)+\omega_{Q}(i) \cdot \operatorname{arbitrary}() \\
P_{t}(i)=P_{\text {initial }}(i)+\omega_{P}(i) \cdot \operatorname{arbitrary}()
\end{array}\right. \\
\text { Location }_{t}=\left\{\begin{array}{c}
\sqrt{Q_{t}^{2}+P_{t}^{2}} \text { if } Q_{\mathrm{i}}+\mathrm{P}_{\mathrm{i}}>0 \\
-\sqrt{Y_{t}^{2}+Z_{t}^{2}} \text { if } \mathrm{Q}_{\mathrm{i}}+\mathrm{P}_{\mathrm{i}}<0
\end{array}\right.
\end{array}\right.
$$

Then the $t_{t h}$ iterations steps are $s t e p_{Q}^{t}$ and $\operatorname{step}_{P}^{t}$, then

$$
\operatorname{step}_{Q}^{t}(i)=Q_{t}(i)-Q_{t-1}(i)(i=1,2, \ldots, N)
$$

Small steps ( small $\left._{\text {steps }}\right)$ and large steps $\left(\right.$ large $\left._{\text {steps }}\right)$ are recorded in $\mathrm{N}$ - dimensional plane

$$
\omega_{t}(i)=\left(\operatorname{large}_{\text {steps }_{t}}(i)-\operatorname{small}_{\text {steps }_{t}}(i)\right) *\left[e^{\frac{\text { step }_{t-1}-(i)-\text { small }_{\text {steps }}(i)}{\text { argestep }_{t}(i)-\text { small }_{\text {steps }}(i)}-1}\right]+\operatorname{small}_{\text {steps }_{t}}(i)
$$

A normal distribution [42-46] is utilized to augment the spread of $\omega_{t}$,

$$
f(q)=\frac{1}{\sqrt{2 \pi}} \exp \left(-\left(q-\omega_{t}\right)^{2}\right)
$$

Gradient compressed progressively by,

$$
\text { Gradient }_{t}(i)=\frac{\text { Number of Melon flies } *\left\|Q_{t}(i)-Q_{t-1}(i)\right\|}{\sum_{i}\left\|Q_{t}(i)\right\|}
$$

Sub swarm (SS) is described as,

$$
S S(i)=\frac{\operatorname{Gradient}(i)}{\sum_{i} \operatorname{Gradient}(i)}
$$

After the completion of definite number of iterations' the melon fly will not fly arbitrarily with the obtained memory so a memory move or progress direction has been applied in the algorithm.

$$
\text { Memory step }{ }_{t}=Q_{t}-Q_{t-1}
$$

Then the exploration process is given by,

$$
\begin{aligned}
& \left\{Q_{t+1}=Q_{t}+\lambda \cdot \text { Memory step } \text { se }_{t} \cdot\right. \text { arbitrary ( ) } \\
& \left\{\begin{array}{l}
P_{t+1}=P_{t}+\lambda \cdot \text { Memory step } \\
t
\end{array}\right. \text { arbitrary () } \\
& \lambda=\lambda_{\text {maximum }} \cdot \exp \left(\log \left(\frac{\lambda_{\text {minimum }}}{\lambda_{\text {maximum }}}\right) \cdot \frac{t}{t_{\text {maximum }}}\right)
\end{aligned}
$$

Previous to iteration $(\varphi)$, the Melon flies will go for collaborative exploration and when iteration number reached the $\varphi$ then Memory move or progress direction will be turned on.

$$
f(q)= \begin{cases}0 & q<\varphi \\ \left\lceil\text { Memory } \cdot 2 \cdot\left(\frac{1}{1+e^{-\frac{y-\varphi}{\varphi}}}-\frac{1}{2}\right)\right] & q \geq \varphi\end{cases}
$$

Begin

Parameters are initialized

Swarm location initialized by 


$$
\left\{\begin{array}{c}
\left\{\begin{array}{c}
Q_{\text {initial }}=\operatorname{arbitrary}(\text { domain }) \\
P_{\text {initial }}=\operatorname{arbitrary}(\text { domain })
\end{array}\right. \\
\text { Location }_{\text {initial stage }}=\left\{\begin{array}{c}
\sqrt{Q_{i}^{2}+P_{i}^{2}} \text { if } Q_{\mathrm{i}}+\mathrm{P}_{\mathrm{i}}>0 \\
-\sqrt{Q_{i}^{2}+P_{i}^{2}} \text { if } \mathrm{Q}_{\mathrm{i}}+\mathrm{P}_{\mathrm{i}}<0
\end{array}\right. \\
\text { Top smell } \text { initial stage }=\text { Fitness }\left(\text { Location }_{\text {initial stage }}\right)
\end{array}\right.
$$

Iteration $=0$

$$
\text { Top smell }=\text { Fitness }\left(J v_{\text {initial stage }}\right)
$$

Repeat

$$
\begin{aligned}
& \text { while iteration }<\varphi \\
& \operatorname{step}_{Q}^{t}(i)=Q_{t}(i)-Q_{t-1}(i)(i=1,2, \ldots, N) \\
& \omega_{t}(i)=\left(\operatorname{large}_{\text {steps }_{t}}(i)-\text { small }_{\text {steps }_{t}}(i)\right) *\left[e^{\frac{\text { step }_{t-1}-(i)-\text { small }_{\text {steps }}(i)}{\text { arge }_{\text {steps }}(i)-\text { small }_{\text {steps }}(i)}-1}\right]+\operatorname{small}_{\text {steps }_{t}}(i) \\
& \left\{\begin{array}{l}
\left\{\begin{array}{l}
Q_{t}(i)=Q_{\text {initial }}(i)+\omega_{Q}(i) \cdot \operatorname{arbitrary}() \\
P_{t}(i)=P_{\text {initial }}(i)+\omega_{P}(i) \cdot \operatorname{arbitrary}()
\end{array}\right. \\
\text { Location }_{t}=\left\{\begin{array}{l}
\sqrt{Q_{t}^{2}+P_{t}^{2}} \text { if } \mathrm{Q}_{\mathrm{i}}+\mathrm{P}_{\mathrm{i}}>0 \\
-\sqrt{Y_{t}^{2}+Z_{t}^{2}} \text { if } \mathrm{Q}_{\mathrm{i}}+\mathrm{P}_{\mathrm{i}}<0
\end{array}\right.
\end{array}\right.
\end{aligned}
$$

End if

End if

$$
\begin{gathered}
\operatorname{Gradient}_{t}(i)=\frac{\text { Number of Melon flies } *\left\|Q_{t}(i)-Q_{t-1}(i)\right\|}{\sum_{i}\left\|Q_{t}(i)\right\|} \\
P(i)=\frac{\text { Gradient }(i)}{\sum_{i} \operatorname{Gradient}(i)} \\
\text { if } \text { arbitrary }<S S(i) \text { then } Q(i)=\operatorname{arbitrary~(domain)~}
\end{gathered}
$$

End

$$
\begin{aligned}
& \text { while iteration }<\varphi \\
& \left\{\begin{array}{l}
Q_{\text {initial }}=\operatorname{arbitrary}(\text { domain }) \\
P_{\text {initial }}=\operatorname{arbitrary}(\text { domain })
\end{array}\right. \\
& \text { Location }_{\text {initial stage }}=\left\{\begin{array}{c}
\sqrt{Q_{i}^{2}+P_{i}^{2}} \text { if } \mathrm{Q}_{\mathrm{i}}+\mathrm{P}_{\mathrm{i}}>0 \\
-\sqrt{Q_{i}^{2}+P_{i}^{2}} \text { if } \mathrm{Q}_{\mathrm{i}}+\mathrm{P}_{\mathrm{i}}<0
\end{array}\right. \\
& \text { Top smell } \text { initial stage }=\text { Fitness }\left(\text { Location }_{\text {initial stage }}\right) \\
& \text { Memory step }_{t}=Q_{t}-Q_{t-1} \\
& Q_{t}(i)=Q_{\text {initial }}(i)+\lambda \cdot \text { Memory } \text { step }_{t} \cdot \operatorname{arbitrary}() i=1,2,3, \ldots, f(y) \\
& Q_{t}(i)=Q_{\text {initial }}(i)+\omega_{y}(i) \cdot \operatorname{arbitrary}() i=f(Q)+1, \ldots, N
\end{aligned}
$$




$$
\begin{gathered}
\text { Location }_{\text {initial stage }}=\left\{\begin{array}{l}
\sqrt{Q_{i}^{2}+P_{i}^{2}} \text { if } \mathrm{Q}_{\mathrm{i}}+\mathrm{P}_{\mathrm{i}}>0 \\
-\sqrt{Q_{i}^{2}+P_{i}^{2}} \text { if } \mathrm{Q}_{\mathrm{i}}+\mathrm{P}_{\mathrm{i}}<0
\end{array}\right. \\
\left\{\begin{array}{r}
{[\text { Top smell, Top index }]=\text { Minimum }(\text { smell })} \\
\left\{\begin{array}{l}
Q_{\text {initial }}=Q(\text { Top } \text { index }) \\
P_{\text {initial }}=P(\text { Top } \text { index })
\end{array}\right.
\end{array}\right.
\end{gathered}
$$

End if

End if

$$
\begin{gathered}
\operatorname{Gradient}_{t}(i)=\frac{\text { Number of Melon flies } *\left\|Q_{t}(i)-Q_{t-1}(i)\right\|}{\sum_{i}\left\|Q_{t}(i)\right\|} \\
S S(i)=\frac{\text { Gradient }(i)}{\sum_{i} \operatorname{Gradient}(i)} \\
\text { if } \text { arbitrary }<S S(i) \text { then } Q(i)=\operatorname{arbitrary}(\text { domain) }
\end{gathered}
$$

End

Until iteration $=$ maximum generation

End

\section{Spontaneous Process Algorithm}

Process of nuclear reaction has been imitated to design Spontaneous Process Algorithm (SPA). In a preserved pot Exploration space is defined and it represents a nucleus which has mass number, charge, location, potential and kinetic energy [11]. Order of operators induce the nucleus (quality of dissimilar condition of reaction) in order to keep away from the local optimum. In exploration space each nucleus signifies variable and solution. Population of $\mathrm{i}$-th nucleus is created by following equation,

$$
H_{i, d}=\text { lower bound }_{d}+\text { arbitrary } \cdot\left(\text { upper bound } d-\text { lowerbound }_{d}\right)(41
$$

Through nuclear fusion neutrons in heated state is produced,

$$
\text { neutron heated state } \mathrm{i}-\operatorname{th}\left(h_{i}\right)=\frac{\left(i-\text { th nucleus }\left(H_{i}\right)+j-\text { th nucleus }\left(H_{j}\right)\right)}{2}
$$

Secondary Fission advocates the discrepancy between existing solution and neutron in heated state to compose in same range. Once arbitrary $\leq$ probability of $\beta$ decay then the development process of Secondary fission, is defined by

$$
\begin{array}{r}
H_{i}^{\text {fission }}=\operatorname{Gaussian}\left(H_{\text {best }}, \sigma_{1}\right)+\left(\operatorname{arbitrary} n \cdot H_{\text {best }}-\text { Mutation factor }(M F) \cdot h e_{i}\right) \\
\sigma_{1}=\left(\frac{\log (\text { existing generation })}{\text { existing generation }}\right) \cdot\left|H_{i}-H_{\text {best }}\right|( \\
M F=\text { round }(\text { arbitrary }+1)
\end{array}
$$

Prime fission is formed by,

$$
\begin{array}{r}
H_{i}^{\text {fission }}=\operatorname{Gaussian}\left(H_{i}, \sigma_{2}\right)+\left(\operatorname{arbitrary} n \cdot H_{\text {best }}-\text { Mutation factor } \cdot h e_{i}\right) \\
\sigma_{2}=\left(\frac{\log (\text { existing generation })}{\text { existing generation }}\right) \cdot\left|H_{\text {arbitrary }}-H_{\text {best }}\right|(47) \\
M F=\text { round }(\text { arbitrary }+2)(48)
\end{array}
$$

Gaussian walk described as,

$$
H_{i}^{\text {fission }}=\operatorname{gaussian}\left(H_{i} \cdot \sigma_{2}\right)(49)
$$

With respect to fitness values various types of nucleus are categorized in appropriate mode in the ionization section. Through uniform distribution probability $\left(P y_{i}\right)$ i-th nucleus defined as, 


$$
P y_{i}=\frac{\text { Level }\left(\text { fitness } Y_{i}^{\text {fitness }}\right)}{\text { entire number of nuclei }}(50)
$$

$\mathrm{d}$ - th variable ionization $H_{i}^{\text {fitness }}$ described as,

$$
\begin{gathered}
H_{i, d}^{i o n}=H_{r 1, d}^{\text {fitness }}+\operatorname{arbitrary} \cdot\left(H_{r 2, d}^{\text {fitness }}-H_{i, d}^{\text {fitness }}\right) \text {, arbitrary } \leq 0.50 \\
H_{i, d}^{i o n}=H_{r 1, d}^{\text {fitness }}-\operatorname{arbitrary} \cdot\left(H_{r 2, d}^{\text {fitness }}-H_{i, d}^{\text {fitness }}\right) \text {, arbitrary }>0.50
\end{gathered}
$$

Slender disturbance in $H_{r 1, d}^{f i t n e s s}$ is included to improve the exploitation as shown below,

$$
\left.H_{i, d}^{\text {ion }}=H_{i, d}^{\text {fitness }}+\text { round (arbitrary) }\right) \cdot\left(H_{\text {worst }, d}^{\text {fitness }}-H_{\text {best }, d}^{\text {fitness }}\right)
$$

i-th ion probability value found by,

$$
p y_{i}=\frac{\text { level }\left(\text { fitnessY } Y_{i}^{\text {ion }}\right)}{\text { whole nuclei }}(54)
$$

Exploration is enhanced by various operators and it replicates the collision and fusion;

$$
H_{i}^{f u s i o n}=H_{i}^{i o n}+\operatorname{arbitrary} \cdot\left(H_{r 1}^{i o n}-H_{b e s t}^{i o n}\right)+\operatorname{arbitrary} \cdot\left(\begin{array}{c}
\left.H_{r 2}^{i o n}-H_{b e s t}^{i o n}\right) \\
\left.H_{r 2}^{i o n}\right)
\end{array}\right)-e^{-\operatorname{normal}\left(H_{r 1}^{i o n}-H_{r 2}^{i o n}\right)} \cdot\left(\begin{array}{l}
H_{r 1}^{i o n}- \\
(55)
\end{array}\right.
$$

Velocity will be decreased whenever fusion does not happened with reference to Coulomb force [47-50] and it defined as,

$$
\begin{array}{r}
H_{i}^{\text {fusion }}=H_{i}^{\text {ion }}-0.50 \cdot\left(\sin (2 \pi . \text { frequency } \cdot g+\pi) \cdot \frac{\text { maximum generation-existing generation }}{\text { maximum generation }}+1\right) \cdot\left(H_{r 1}^{\text {ion }}-\right. \\
\left.H_{r 2}^{\text {ion }}\right) \text { arbitrary }>0.50(56)
\end{array}
$$

Levy flight is a rank of non-Gaussian random procedure [42-46] and allocation by $L(s) \sim|s|^{-1-\beta}$ where $0<\beta$ $<2$ is an index. Scientifically defined as,

$$
\begin{gathered}
L y(s, \gamma, \mu)=\left\{\begin{array}{r}
\sqrt{\frac{\gamma}{2 \pi}} \\
0 \quad \text { if } s \leq 0
\end{array} \quad \exp \left[-\frac{\gamma}{2(s-\mu)}\right] \frac{1}{(s-\mu)^{3 / 2}} \quad \text { if } 0<\mu<s<\infty\right. \\
\quad F y(k)=\exp \left[-\alpha|k|^{\beta}\right], 0<\beta \leq 2, \\
s y=\frac{u}{|v|^{\frac{1}{\beta}}} \\
H^{t+1}=H^{t}+\operatorname{arbitrary}(\operatorname{size}(D)) \oplus \operatorname{Levy}(\beta) \sim 0.01 \frac{u}{|v|^{1 / \beta}}\left(H_{j}^{t}-g b\right)
\end{gathered}
$$

Where $H_{j}^{t}$ is the $H^{t}$ solution vector at iteration "t", "u" is an arbitrary parameter which kowtow to a uniform distribution, $\oplus$ is the dot product for entry wise multiplications. And it takes three values 1,0 , and -1 . And in Equation (60) the combination is to say, to get rid of local minima and perk up global search ability are guaranteed by means of this combination.

$$
\begin{array}{r}
u \sim N\left(0, \sigma_{u}^{2}\right) \quad v \sim N\left(0, \sigma_{v}^{2}\right)(61) \\
\sigma_{u}=\left\{\frac{\Gamma(1+\beta) \sin (\pi \beta / 2)}{\Gamma[(1+\beta) / 2] \beta 2^{(\beta-1) / 2}}\right\}^{1 / \beta}, \sigma_{v}=1(62) \\
\operatorname{Levy}(H)=0.01 \times \frac{u \times \sigma}{|v| \frac{1}{\beta}}(63) \\
H_{i, d}^{\text {ion }}=H_{i, d}^{\text {Fission }}+(\alpha \oplus \text { Levy }(\beta))_{d} \cdot\left(H_{i, d}^{\text {fission }}-H_{\text {best }, d}^{\text {fission }}\right)(64) \\
H_{i, d}^{\text {ion }}=H_{i, d}^{\text {Fission }}+(\alpha \oplus \text { Levy }(\beta))_{d} \cdot\left(\text { upper bound }{ }_{d}-\text { lowerbound }_{d}\right)(65)
\end{array}
$$




$$
H_{i}^{\text {fusion }}=H_{i}^{\text {ion }}+(\alpha \oplus \operatorname{Levy}(\beta)) \oplus \cdot\left(H_{i}^{\text {ion }}-H_{\text {best }}^{\text {ion }}\right)
$$

Periphery management done by,

$$
H_{i, d}=\text { lowerbound }_{d}+\text { arbitrary } \cdot\left(\text { upper bound } d-\text { lowerbound }_{d}\right)
$$

a. Begin

b. Creation of Population by $H_{i, d}=$ lower bound $_{d}+$ arbitrary $\cdot\left(\right.$ upper bound $d_{d}-$ lowerbound $\left._{d}\right)$

c. Fitness function of the population is created by

d. While (existing generation < maximum iteration) do

e. existing generation $=$ existing generation +1

f. For $i=1$ to $\mathrm{N} d o$

g. Computation of neutron in heated status is done by

$$
\text { neutron heated state } \mathrm{i}-\operatorname{th}\left(h e_{i}\right)=\frac{\left(i-\text { th nucleus }\left(H_{i}\right)+j-\text { th nucleus }\left(H_{j}\right)\right)}{2}
$$

h. Population updated by

$$
\begin{gathered}
H_{i}^{\text {fission }}=\operatorname{Gaussian}\left(H_{\text {best }}, \sigma_{1}\right)+\left(\operatorname{arbitrary} n \cdot H_{\text {best }}-\text { Mutation factor }(M F) \cdot h e_{i}\right) \\
\sigma_{1}=\left(\frac{\log (\text { existing generation })}{\text { existing generation }}\right) \cdot\left|H_{i}-H_{\text {best }}\right| \\
M F=\text { round }(\text { arbitrary }+1)
\end{gathered}
$$

i. Fitness value is calculated for $H_{i}^{\text {fitness }}$, then frontier conditions are verified

j. $H_{i}^{\text {fitness }}$ and $H_{i}^{\text {generation }}$ are updated

k. Computed Probability Pr $_{i}=\frac{\text { level }\left(\text { fitness } H_{i}^{\text {fitness }}\right)}{\text { entire nuclei }}$

I. For $i=1$ to $N$ do

m. For $d=1$ to $N$ do

n. levy flight applied by

o. $H_{i, d}^{\text {ion }}=H_{i, d}^{\text {Fission }}+(\alpha \oplus \text { Levy }(\beta))_{d} \cdot\left(H_{i, d}^{\text {fission }}-H_{\text {best }, d}^{\text {fision }}\right)$

p. $\quad H_{i, d}^{\text {ion }}=H_{i, d}^{\text {Fission }}+(\alpha \oplus \text { Levy }(\beta))_{d} \cdot\left(\right.$ upper bound blowerbound $\left._{d}\right)$

q. $H_{i}^{\text {fusion }}=H_{i}^{\text {ion }}+(\alpha \oplus \operatorname{Levy}(\beta)) \oplus \cdot\left(H_{i}^{\text {ion }}-H_{\text {best }}^{\text {ion }}\right)$

r. Updating of lon states is done through,

$$
\begin{aligned}
& H_{i, d}^{i o n}=H_{r 1, d}^{\text {fitness }}+\operatorname{arbitrary} \cdot\left(H_{r 2, d}^{\text {fitness }}-H_{i, d}^{\text {fitness }}\right) \text {, arbitrary } \leq 0.50 \\
& H_{i, d}^{\text {ion }}=H_{r 1, d}^{\text {fitness }}-\operatorname{arbitrary} \cdot\left(H_{r 2, d}^{\text {fitness }}-H_{i, d}^{f i t n e s s}\right) \text {, arbitrary }>0.50
\end{aligned}
$$

s. Slender disturbance in $\mathrm{Y}_{\mathrm{r} 1, \mathrm{~d}}^{\text {fitness }}$ is included to improve the exploitation as shown below,

$$
H_{i, d}^{\text {ion }}=H_{i, d}^{\text {fitness }}+\text { round }(\text { arbitrary }) \cdot\left(H_{\text {worst }, d}^{\text {fitness }}-H_{\text {best }, d}^{\text {fitness }}\right)
$$

t. End for

u. fitness value of $H_{i}^{\text {ion }}$ compute the calculated and frontier conditions are verified

v. $H_{i}^{\text {fitness }}$ and $H_{i}^{\text {ion }}$ are updated

w. End for

x. calculate probability

$p y_{i}=\frac{\text { level }\left(\text { fitness } H_{i}^{\text {ion }}\right)}{\text { entire nuclei }}$

y. For $i=1$ to $N$ do

z. levy flight applied by

$H_{i}^{\text {fusion }}=H_{i}^{\text {ion }}+(\alpha \oplus \operatorname{Levy}(\beta)) \oplus \cdot\left(H_{i}^{\text {ion }}-H_{\text {best }}^{\text {ion }}\right)$

aa. population of fusion updated by,

$H_{i}^{f u s i o n}=H_{i}^{i o n}+\operatorname{arbitrary} \cdot\left(H_{r 1}^{i o n}-H_{\text {best }}^{i o n}\right)+\operatorname{arbitrary} \cdot\left(H_{r 2}^{i o n}-H_{\text {best }}^{i o n}\right)-e^{-\operatorname{normal}\left(H_{r 1}^{i o n}-H_{r 2}^{i o n}\right)} \cdot\left(H_{r 1}^{i o n}-H_{r 2}^{i o n}\right)$ 


$$
\begin{aligned}
H_{i}^{\text {fusion }}=H_{i}^{\text {ion }} & -0.50 \\
& \cdot\left(\sin (2 \pi \cdot \text { frequency } \cdot g+\pi) \cdot \frac{\text { maximum generation }- \text { existing generation }}{\text { maximum generation }}+1\right) \cdot\left(H_{r 1}^{\text {ion }}\right. \\
& \left.-H_{r 2}^{i o n}\right) \text { arbitrary }>0.50 \\
H_{i}^{\text {fusion }}=H_{i}^{i o n} & -0.50 \cdot\left(\sin (2 \pi . \text { frequency } \cdot g+\pi) \cdot \frac{\text { existing generation }}{\text { maximum generation }}+1\right) \cdot\left(H_{r 1}^{\text {ion }}-H_{r 2}^{\text {ion }}\right) \text { arbitrary } \\
\leq & 0.50
\end{aligned}
$$

bb. fitness value of $H_{i}^{\text {fusion }}$ compute the calculated and frontier conditions are verified

cc. $H_{i}^{\text {fusion }}$ and $H_{i}^{\text {ion }}$ are updated

dd. End while

ee. Output; most excellent solution

\section{RESULTS}

With considering $L$ - index Melon Fly Optimization (MFO) Algorithm and Spontaneous Process Algorithm (SPA) evaluated in IEEE 30 bus system [21]. Table 1 shows the optimized values. Tables 2 to 4 give the comparison of parameters. Figure 1 shows the real power loss comparison. Figures 2 and 3 show the voltage deviation and stability index. Figure 4 shows the power loss evaluation and Figure 5 shows the convergence characteristics

Table 1.Optimal solutions of the proposed algorithms

\begin{tabular}{ccccccc}
\hline Parameter & $\begin{array}{c}\text { Real Power } \\
\text { loss } \\
\text { (MFO) }\end{array}$ & $\begin{array}{c}\text { Real Power } \\
\text { loss } \\
\text { (SPA) }\end{array}$ & $\begin{array}{c}\text { Voltage } \\
\text { Deviation } \\
\text { (MFO) }\end{array}$ & $\begin{array}{c}\text { Voltage } \\
\text { Deviation } \\
\text { (SPA) }\end{array}$ & $\begin{array}{c}\text { Voltage } \\
\text { Stability Index } \\
\text { (MFO) }\end{array}$ & $\begin{array}{c}\text { Voltage } \\
\text { Stability Index } \\
\text { (SPA) }\end{array}$ \\
\hline VG1 & 1.1002 & 1.1003 & 1.0056 & 1.0057 & 1.0804 & 1.0801 \\
VG2 & 1.0953 & 1.0952 & 1.0014 & 1.0013 & 1.0532 & 1.0530 \\
VG5 & 1.0753 & 1.0751 & 1.0170 & 1.0173 & 1.0731 & 1.0730 \\
VG8 & 1.0762 & 1.0753 & 1.0125 & 1.0127 & 1.0089 & 1.0086 \\
VG11 & 1.0870 & 1.0872 & 1.0323 & 1.0324 & 1.0800 & 1.0803 \\
VG13 & 1.0990 & 1.0991 & 1.0234 & 1.0230 & 1.0855 & 1.0857 \\
T1 & 1.0500 & 1.0501 & 1.0500 & 1.0500 & 0.9000 & 0.9000 \\
T2 & 0.9200 & 0.9201 & 0.9000 & 0.9000 & 0.9000 & 0.9000 \\
T3 & 1.0100 & 1.0102 & 1.0000 & 1.0001 & 0.9000 & 0.9000 \\
T4 & 0.9800 & 0.9803 & 0.9700 & 0.9701 & 0.9000 & 0.9000 \\
Qc1 & 5.0000 & 5.0000 & 4.0000 & 4.0000 & 5.0000 & 5.0000 \\
Qc2 & 5.0000 & 5.0000 & 2.0000 & 2.0000 & 5.0000 & 5.0000 \\
Qc3 & 5.0000 & 5.0000 & 4.0000 & 4.0000 & 0.0000 & 0.0000 \\
Qc4 & 5.0000 & 5.0000 & 3.0000 & 3.0000 & 0.0000 & 0.0000 \\
Qc5 & 3.0000 & 3.0000 & 5.0000 & 5.0000 & 5.0000 & 5.0000 \\
Qc6 & 5.0000 & 5.0000 & 3.0000 & 3.0000 & 3.0000 & 3.0000 \\
Qc7 & 3.0000 & 3.0000 & 5.0000 & 5.0000 & 5.0000 & 5.0000 \\
Qc8 & 4.0000 & 4.0000 & 5.0000 & 5.0000 & 5.0000 & 5.0000 \\
Qc9 & 2.0000 & 2.0000 & 3.0000 & 3.0000 & 1.0000 & 1.0000 \\
\hline
\end{tabular}


Table 2. Comparison of total power loss for IEEE 30 bus system

\begin{tabular}{lc}
\hline Method & Power loss (MW) \\
\hline Hybrid PSO-TS [14] & 4.5213 \\
TS [14] & 4.6862 \\
Basic PSO [14] & 4.6862 \\
ALO [15] & 4.5900 \\
QO-TLBO [15] & 4.5594 \\
TLBO [15] & 4.5629 \\
Standard GA [16] & 4.9408 \\
S.PSO [16] & 4.9239 \\
HAS [16] & 4.9059 \\
S-FS [17] & 4.5777 \\
IS-FS [17] & 4.5142 \\
SFS [19] & 4.5275 \\
MFO & 4.5082 \\
SPA & 4.5094 \\
\hline
\end{tabular}

\section{Factual Power loss (MW)}

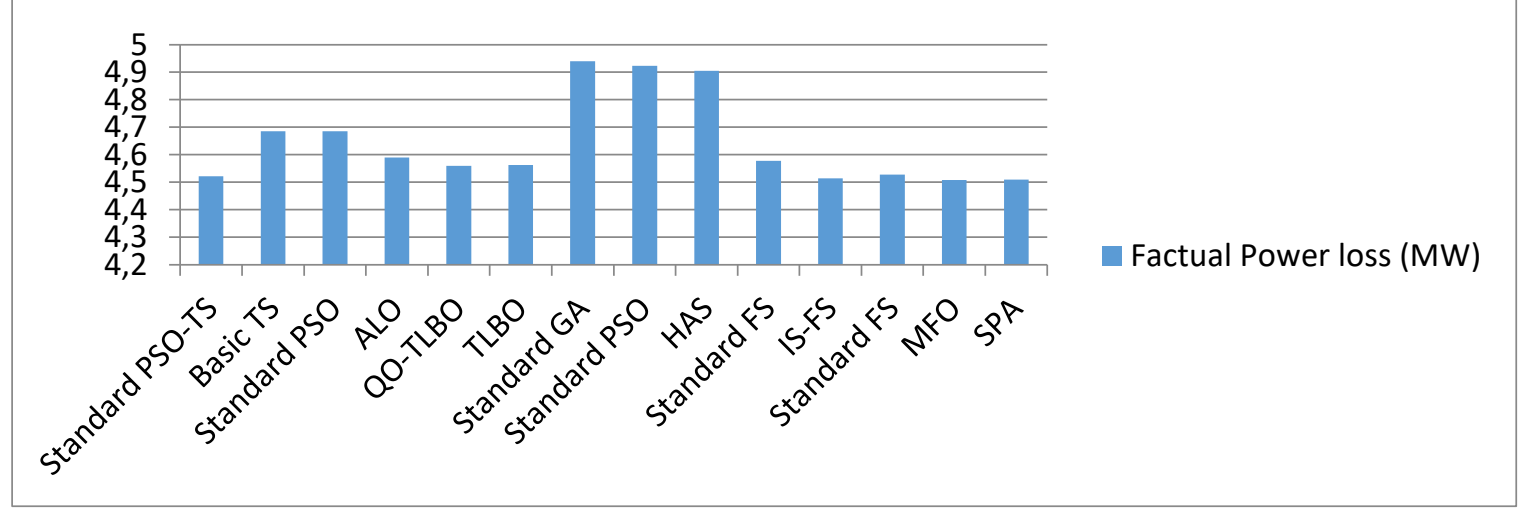

Figure 1. Comparison of real power loss

Table 3. Comparison of voltage deviation for IEEE 30 bus system.

\begin{tabular}{lc}
\hline Method & Voltage deviation (PU) \\
\hline BPSO-TVIW [18] & 0.1038 \\
BPSO-TVAC [18] & 0.2064 \\
SPSO-TVAC [18] & 0.1354 \\
BPSO-CF [18] & 0.1287 \\
PG-PSO [18] & 0.1202 \\
SWT-PSO [18] & 0.1614 \\
PGSWT-PSO [18] & 0.1539 \\
MPG-PSO [18] & 0.0892 \\
QO-TLBO [15] & 0.0856 \\
TLBO [15] & 0.0913 \\
S-FS [17] & 0.1220 \\
ISFS [17] & 0.0890 \\
SFS [19] & 0.0877 \\
MFO & 0.0873 \\
SPA & 0.0870 \\
\hline
\end{tabular}




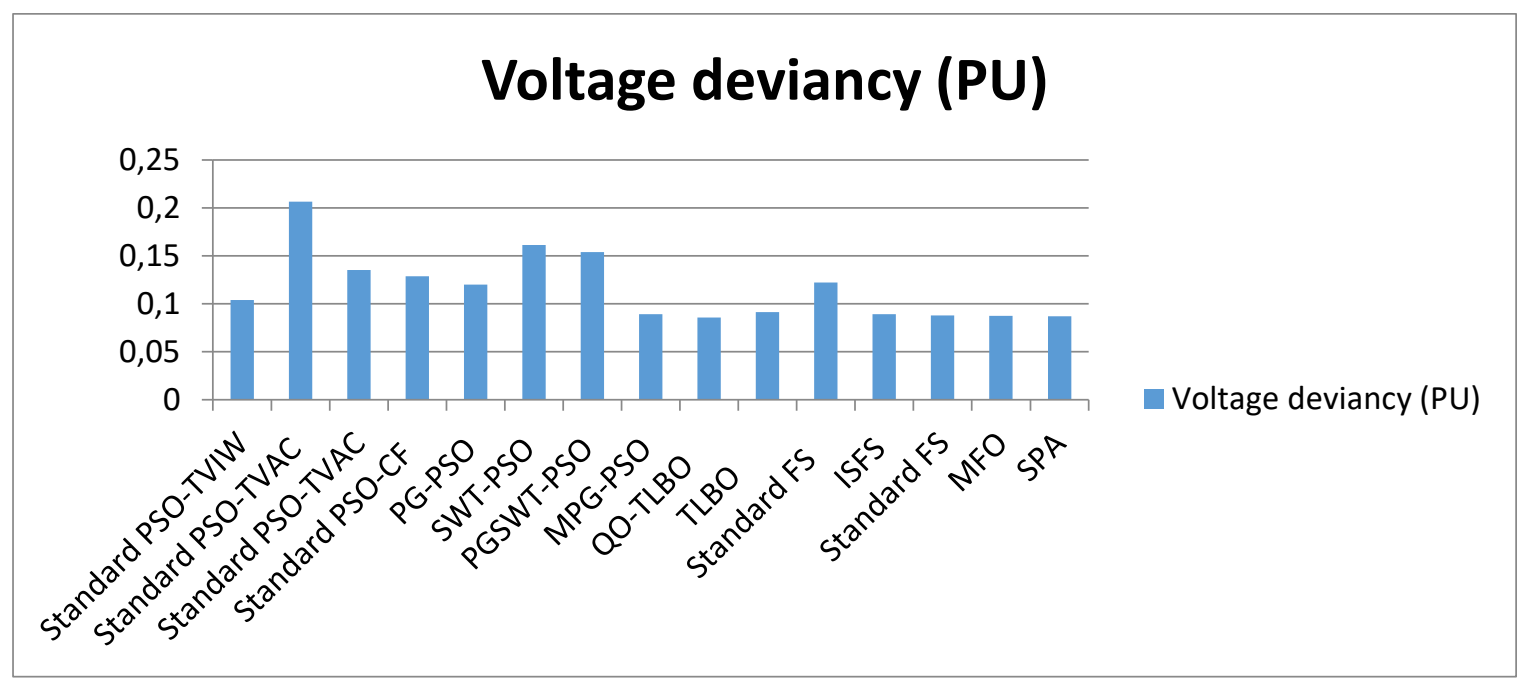

Figure 2. Comparison of Voltage deviation.

Table 4. Comparison of Voltage Stability Index for IEEE 30 bus system.

\begin{tabular}{lc}
\hline \multicolumn{1}{c}{ Method } & L-index (PU) \\
\hline BPSO-TVIW [18] & 0.1258 \\
BPSO-TVAC[18] & 0.1499 \\
SPSO-TVAC[18] & 0.1271 \\
BPSO-CF [18] & 0.1261 \\
PG-PSO [18] & 0.1264 \\
SWT-PSO [18] & 0.1488 \\
PGSW-PSO [18] & 0.1394 \\
MPG-PSO [18] & 0.1241 \\
QO-TLBO [15] & 0.1191 \\
TLBO [15] & 0.1180 \\
ALO[14] & 0.1161 \\
ABC [14] & 0.1161 \\
GWO [14] & 0.1242 \\
BA [14] & 0.1252 \\
S-FS [17] & 0.1252 \\
IS-FS [17] & 0.1245 \\
SFS [19] & 0.1007 \\
MFO & 0.1006 \\
SPA & 0.1004 \\
\hline
\end{tabular}

\section{Voltage constancy (PU)}

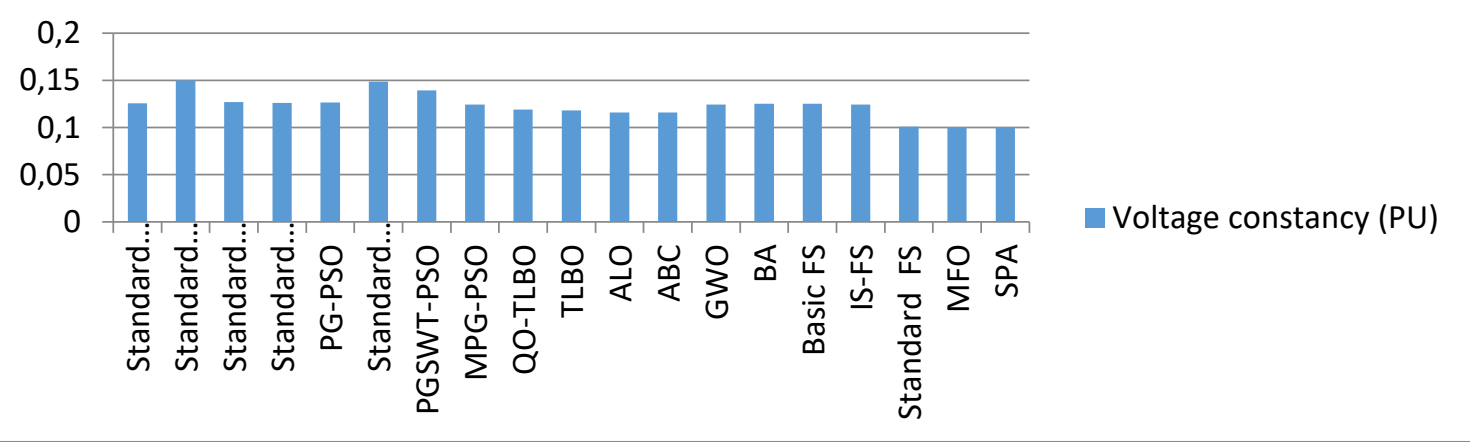

Figure 3. Comparison of voltage stability index

Then Melon Fly Optimization (MFO) Algorithm and Spontaneous Process Algorithm (SPA) verified in IEEE 30, bus system [51] without L-index. Comparisons of results are presented in Table 5. Comparison of 
loss has been done with other algorithms - particle swarm optimization (PSO), Modified PSO, Evolutionary programming, self -adaptive real coded genetic algorithm. Projected algorithms very effectively reduced the power loss. Percentage of real power loss reduction has been improved.

Table 5.Loss comparison with reference to IEEE -30 system

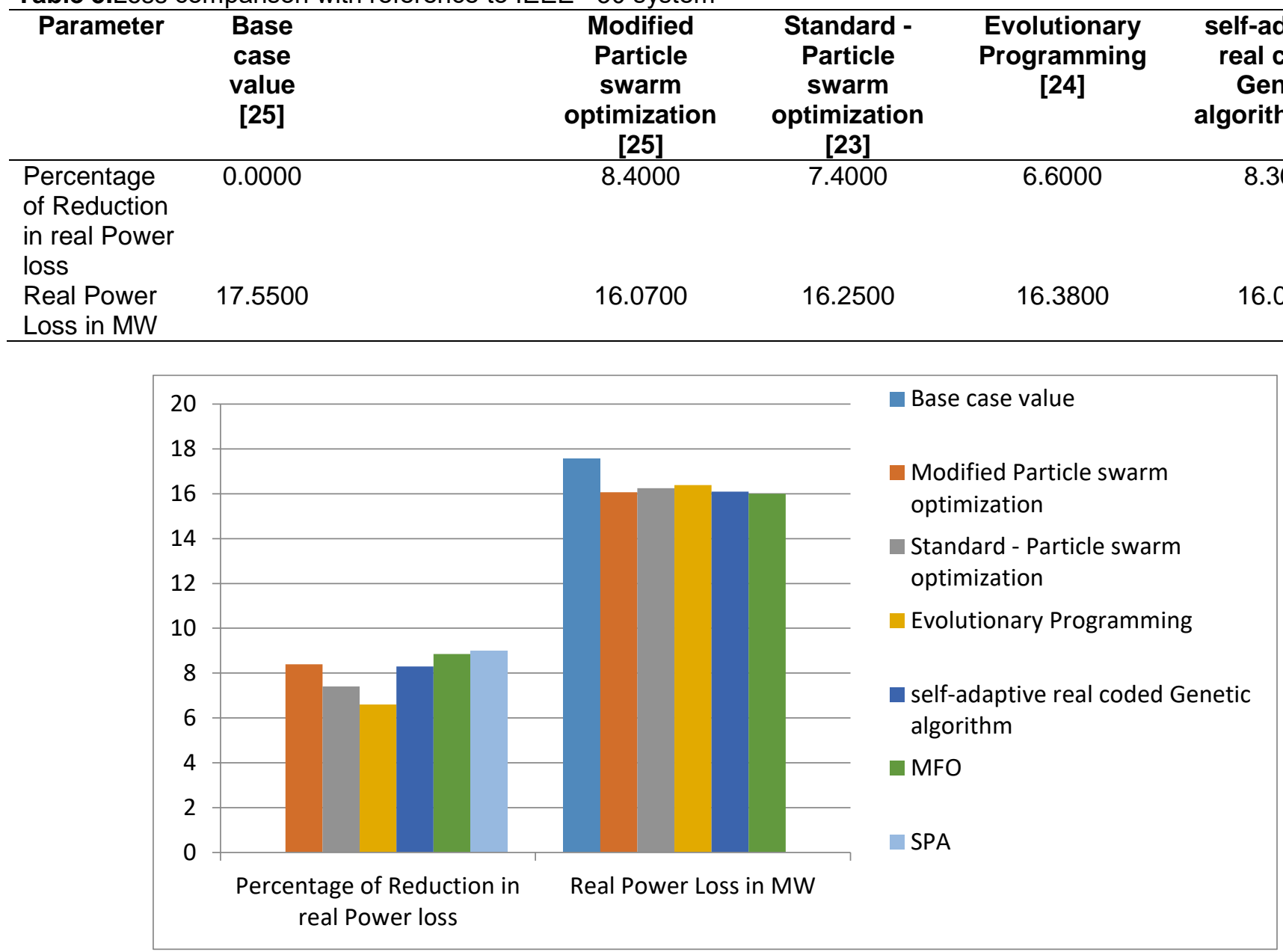

Figure 4. Comparison of Real Power Loss between methodologies (Tested in IEEE 30 bus system)

Table 6 shows the convergence characteristics of the proposed Melon Fly Optimization (MFO) Algorithm and Spontaneous Process Algorithm (SPA).

Table 6. Convergence characteristics.

\begin{tabular}{ccccccc}
\hline $\begin{array}{l}\text { IEEE 30 } \\
\text { Bus }\end{array}$ & $\begin{array}{c}\text { Real power Loss } \\
\text { in MW(With L- } \\
\text { system }\end{array}$ & $\begin{array}{c}\text { Real power Loss } \\
\text { in MW (without L- } \\
\text { index) }\end{array}$ & $\begin{array}{c}\text { Time in Sec } \\
\text { (with L- } \\
\text { index) }\end{array}$ & $\begin{array}{c}\text { Time in sec ( } \\
\text { without L- } \\
\text { index) }\end{array}$ & $\begin{array}{c}\text { Number of } \\
\text { iterations } \\
\text { (with L- } \\
\text { index) }\end{array}$ & $\begin{array}{c}\text { Number of } \\
\text { iterations } \\
\text { (without L- } \\
\text { index) }\end{array}$ \\
\hline MFO & 4.5082 & 15.996 & 18.64 & 14.80 & 26 & 23 \\
SPA & 4.5094 & 15.971 & 18.69 & 14.82 & 28 & 21 \\
\hline
\end{tabular}




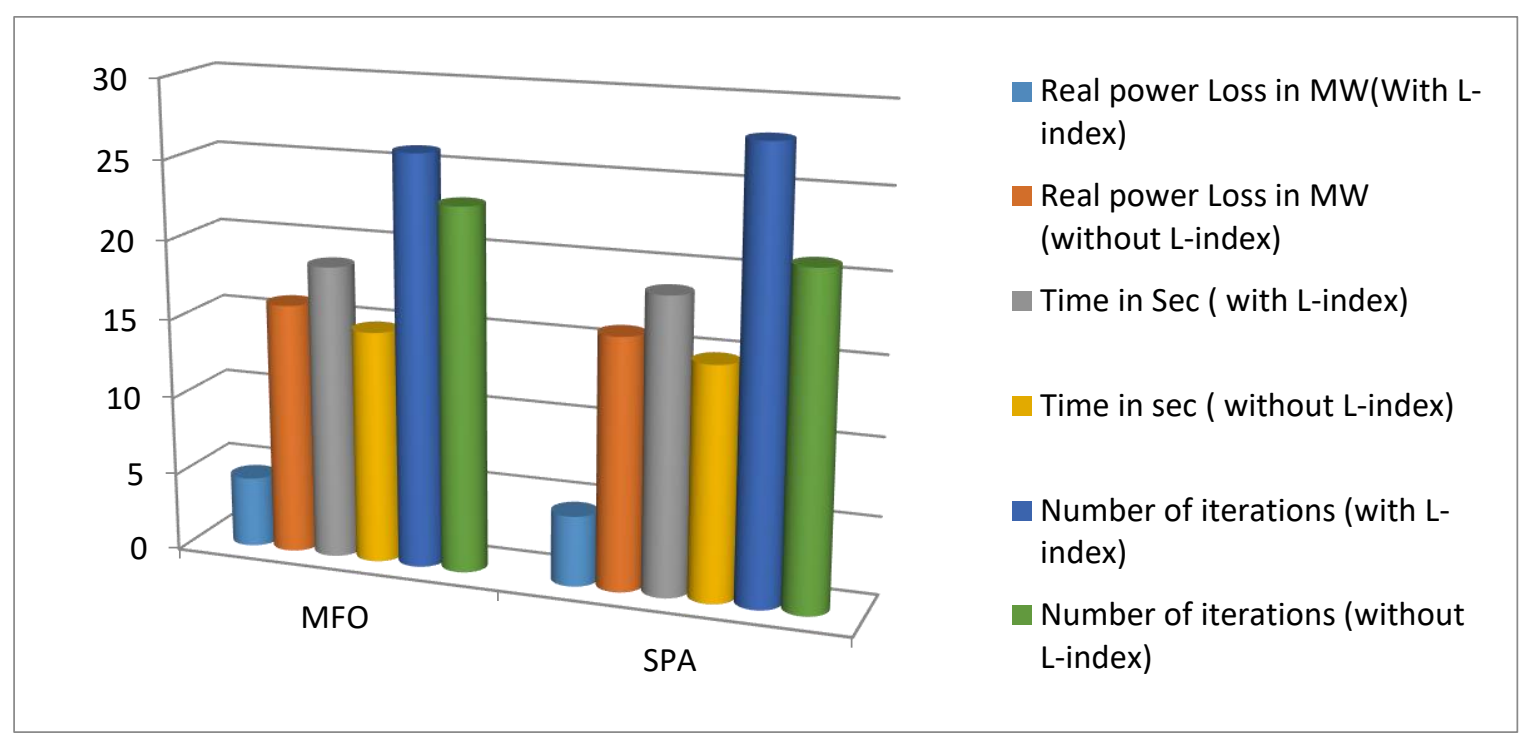

Figure 5. Convergence characteristics of MFO and SPA.

\section{DISCUSSION}

Both Melon Fly Optimization (MFO) Algorithm and Spontaneous Process Algorithm (SPA) reduced the power loss efficiently. Voltage stability augmentation with voltage deviation minimization has been achieved. Comparison of results are done with Hybrid PSO-Tabu search, Ant lion, quasi-oppositional teaching learning based, improved stochastic fractal search optimization algorithm, harmony search, improved pseudogradient search particle swarm optimization and cuckoo search algorithm.

\section{CONCLUSION}

Key aim of this paper is real power loss reduction with voltage stability enhancement. Voltage deviation minimization is additional objective of the paper. Both Melon Fly Optimization (MFO) Algorithm and Spontaneous Process Algorithm (SPA) reduced the power loss effectively. In MFO, after the completion of specific number of iterations' the melon fly will not fly capriciously with the acquired memory, so a memory shift or advancement direction has been applied in the MFO algorithm. In SPA approach with respect to fitness values different types of nucleus are classified in pertinent mode in the ionization section. With considering L - index Proposed Melon Fly Optimization (MFO) Algorithm and Spontaneous Process Algorithm (SPA) evaluated in IEEE 30, bus system with and without considering voltage stability enhancement index. Comparison of simulation results done with Hybrid PSO-Tabu search (PSO-TS), Ant lion (ALO), quasioppositional teaching learning based (QOTBO), improved stochastic fractal search optimization algorithm (ISFS), harmony search (HS), improved pseudo-gradient search particle swarm optimization and cuckoo search algorithm. Then for without considering voltage stability the comparison done with particle swarm optimization (PSO), Modified PSO, Evolutionary programming, self - adaptive real coded genetic algorithm. Projected algorithms very effectively reduced the power loss. Percentage of real power loss reduction has been improved. In MFO Real power Loss in MW (With L-index) value obtained is 4.5082 and value of Real power Loss in MW (without L-index) is 15.996. Time (S) required for reaching the solution (with L-index) is 18.64and Time (s) for (without L-index) is 14.80. Then number of iterations (with L-index) is 26 and number of iterations (without L-index) is 23. In SPA Real power Loss in MW (With L-index) value obtained is 4.5094 and value of Real power Loss in MW (without L-index) is 15.971. Time (S) required for reaching the solution (with L-index) is 18.69and Time (s) for (without L-index) is 14.82. Then number of iterations (with L-index) is 28 and number of iterations (without L-index) is 21 . Percentage of the power loss reduction for MFO is $8.85 \%$ and for SPA is $8.99 \%$. So it's well understood that both Melon Fly Optimization (MFO) Algorithm and Spontaneous Process Algorithm (SPA) performed well with respect to the given power loss reduction problem. 


\section{Scope of future work}

In future Both the Melon Fly Optimization (MFO) Algorithm and Spontaneous Process Algorithm (SPA) can be applied to large system (IEEE 300 bus system). Then sequentially it can be applied to practical systems. Further the algorithms can be enhanced and applied to other power system optimization problems (Real and reactive power dispatch problem, Economic dispatch problem and Unit commitment problem).

Funding: This research received no external funding.

Acknowledgments: NIL.

Conflicts of Interest: The authors declare no conflict of interest.

\section{REFERENCES}

1. LeeK. Fuel-cost minimization for both real and reactive-power dispatches. Proceedings Generation, Transmission and Distribution Conference. 1984 Jan;131(3):85-93.

2. DeebN. An efficient technique for reactive power dispatch using a revised linear programming approach. IEEE Trans. Power Systems. 1998 Feb; 15(2): 121-34.

3. BjelogrlicM. Application of Newton's optimal power flow in voltage/reactive power control. IEEE Trans. Power Systems. 1990 June;5(4):1447-54.

4. GranvilleS.Optimal reactive dispatch through interior point methods. IEEE Trans. Power Systems. 1994 Jan; 9(1):136-46.

5. Grudinin, N. Reactive power optimization using successive quadratic programming method. IEEE Trans. Power Systems. 1998 June; 13(4):1219-25.

6. Mavaddat N, Michailidou K, Dennis J, Lush M, Fachal L, Lee A, et al. Polygenic Risk Scores for Prediction of Breast Cancer and Breast Cancer Subtypes. Am J Hum Genet. 2019;104(1):21-34.

7. Kanimozhi U, Ganapathy S, Manjula D, Kannan A. An Intelligent Risk Prediction System for Breast Cancer Using Fuzzy Temporal Rules. Natl AcadSci Lett. 2019;42(3):227-32.

8. Gupta K, Janghel R. Dimensionality reduction-based breast cancer classification using machine learning. In: Advances in Intelligent Systems and Computing. 2019;798:133-46.

9. Sangaiah I, Vincent Antony Kumar A. Improving medical diagnosis performance using hybrid feature selection via relieff and entropy based genetic search (RF-EGA) approach: application to breast cancer prediction. Cluster Comput. 2019;22(s3):6899-906.

10. Park JV, Park SJ, Yoo JS. Finding characteristics of exceptional breast cancer subpopulations using subgroup mining and statistical test. Expert Syst Appl. 2019;118:553-62.

11. Wei,Huang,Wang,HanY. Li. Nuclear Reaction Optimization: A Novel and Powerful Physics-Based Algorithm for Global Optimization.IEEE Access, 2019 May; 7(1):66084-66109.

12. Illinois Center for a Smarter Electric Grid (ICSEG). Available online: https://icseg.iti.illinois.edu/ieee-30-bussystem/ (accessed on 25 February 2019).

13. ZhangAC, Sanderson. JADE: adaptive differential evolution with optional external archive. IEEE Trans EvolCompt, 2009 June; 13(5):945-58.

14. Mouassa S, Bouktir T, Salhi A. Ant lion optimizer for solving optimal reactive power dispatch problem in power systems. Eng, Sci and Tech, 2017 June; 20(3):885-95.

15. Mandal, B. Optimal reactive power dispatch using quasi-oppositional teaching learning based optimization. Elec. Power. Sys. 2013 Oct; 53(1): 123-34.

16. Khazali, H. Optimal reactive power dispatch based on harmony search algorithm. Elec. Power Sys. 2011 Jan 33(3):684-92.

17. Tran, H. Finding optimal reactive power dispatch solutions by using a novel improved stochastic fractal search optimization algorithm. Tele.Control, 2019 Jan ;17(5): 2517-26.

18. PolprasertJ. Optimal reactive power dispatch using improved pseudo-gradient search particle swarm optimization. Elec. Sys. 2016 July; 44(5):518-32.

19. ThanhL. Optimal Reactive Power Flow for Large-Scale Power Systems Using an Effective Metaheuristic Algorithm. Hindawi. 2020 Jan;20(1):1-11.

20. Thanh Long Duong, Minh Quan Duong, Van-DucPhan, Thang TrungNguyen.Optimal Reactive Power Flow for Large-Scale Power Systems Using an Effective Metaheuristic Algorithm. Hindawi, 2020 May; 1(1):1-11.

21. MATPOWER 4.1 IEEE 30-bus and 118-bus test system, [Internet]. 2019. [cited 2019 Sep 02]. Available from: http://www.pserc.cornell.edu/matpower.

22. Dai, Chen, Zhu, Zhang. Seeker optimization algorithm for optimal reactive power dispatch. IEEE Trans. Power Systems, 2009 May; 24(3): 1218-31. 
23. Subbaraj P, Rajnarayan PN. Optimal reactive power dispatch using self-adaptive real coded Genetic algorithm. Elec.Power Sys. 2009 Dec; 79(2): 374-38.

24. Pandya S, Roy R. "Particle swarm optimization based optimal reactive power dispatch" Proceeding of the IEEE International Conference on Electrical, Computer and Communication Technologies (ICECCT); 2015. March 5-7; Coimbatore, India: IEEE; 2006.

25. Ali Nasser Hussain, Ali Abdulabbas Abdullah and Omar Muhammed Neda. Modified Particle Swarm Optimization for Solution of Reactive Power Dispatch. App. Sci., Eng. Tech. 2018 Jan; 15(8): 316-27.

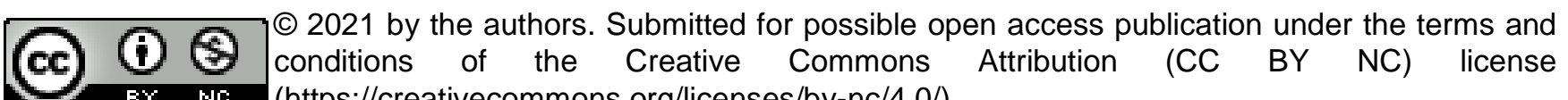
EY NC (https://creativecommons.org/licenses/by-nc/4.0/). 